TRANSACTIONS OF THE

AMERICAN MATHEMATICAL SOCIETY

Volume 349, Number 5, May 1997, Pages 1973-1992

S $0002-9947(97) 01734-0$

\title{
PARTIAL REGULARITY OF SOLUTIONS TO A CLASS OF DEGENERATE SYSTEMS
}

\author{
XIANGSHENG XU
}

Abstract. We consider the system $\frac{\partial u}{\partial t}-\Delta u=\sigma(u)|\nabla \varphi|^{2}, \operatorname{div}(\sigma(u) \nabla \varphi)=$ 0 in $Q_{T} \equiv \Omega \times(0, T]$ coupled with suitable initial-boundary conditions, where $\Omega$ is a bounded domain in $\mathbf{R}^{N}$ with smooth boundary and $\sigma(u)$ is a continuous and positive function of $u$. Our main result is that under some conditions on $\sigma$ there exists a relatively open subset $Q_{0}$ of $Q_{T}$ such that $u$ is locally Hölder continuous on $Q_{0}$, the interior of $Q_{T} \backslash Q_{0}$ is empty, and $u$ is essentially bounded on $Q_{T} \backslash Q_{0}$.

\section{INTRODUCTION}

In this paper we obtain several results concerning the regularity of solutions to the system

$$
\begin{array}{cc}
\frac{\partial u}{\partial t}-\Delta u=\sigma(u)|\nabla \varphi|^{2} \quad \text { in } \quad & Q_{T} \equiv \Omega \times(0, T] \\
\operatorname{div}(\sigma(u) \nabla \varphi)=0 \quad \text { in } \quad Q_{T},
\end{array}
$$

where $\Omega$ is a bounded domain in $\mathbf{R}^{N}$ with smooth boundary $\partial \Omega, T>0$, and $\sigma \in C(\mathbf{R})$ is positive. In [SSX], this system is proposed as a model for a conductor in which both heat conduction and electrical conduction take place. Then $u$ is the temperature of the conductor, and $\varphi$ the electrical potential. The first equation describes the diffusion of heat, while the second equation represents the conservation of electrical charges. The term $\sigma(u)$ is the temperature-dependent electrical conductivity. Its precise form is determined by the particular physical situation one has in mind. See $[\mathrm{XA}]$ for the expression for $\sigma(u)$ in the microsensor applications.

To complete the problem, the system needs to be coupled with suitable initialboundary conditions. Here we impose the following conditions:

$$
\begin{array}{r}
u=\bar{u} \quad \text { on } \quad S_{T} \equiv \partial \Omega \times(0, T], \\
\varphi=\bar{\varphi} \quad \text { on } \quad S_{T}, \\
u(x, 0)=u_{0}(x) \quad \text { on } \quad \Omega .
\end{array}
$$

Here, $\bar{u}, \bar{\varphi}, u_{0}$ satisfy certain conditions to be specified later.

Received by the editors September 26, 1994 and, in revised form, November 27, 1995.

1991 Mathematics Subject Classification. Primary 35B65, 35K65.

Key words and phrases. Partial regularity, degenerate systems.

This work was supported in part by an NSF grant (DMS942448). 
We are interested in the question of how different assumptions on $\sigma$ may affect the regularity of solutions of the problem. If $\sigma \in C^{1}(\mathbf{R})$ is such that

$$
m \leq \sigma(s) \leq M \quad \text { on } \quad \mathbf{R}
$$

for some $m, M \in(0, \infty)$, a result of [YL, Y] indicates that $u \in C^{\alpha, \frac{\alpha}{2}}\left(\bar{Q}_{T}\right)$ for some $\alpha \in(0,1)$. This immediately triggers a bootstrap process which yields higher regularity for $u$ and $\varphi$ if $\sigma$ is smooth enough. The proof in [YL] relies upon the single-layer potential theory. As a by-product of our development here we give a new proof of this result which seems to exhibit a new perspective from which to view the system. We are mainly concerned with the case where $\sigma$ is bounded above and satisfies

$$
\lim _{\tau \rightarrow 0} \frac{\sigma(s+\tau)}{\sigma(s)}=1 \quad \text { uniformly on } \mathbf{R},
$$

and

$$
\lim _{s \rightarrow \infty} \sigma(s)=0 .
$$

The conditions here are rather general $[\mathrm{C}]$. For example, exponential functions of the form $\sigma(s)=e^{-\alpha|s|}, \alpha>0$, satisfy (1.2) and (1.3). In this generality, we do not expect $u$ to be bounded. If $u$ is unbounded, the system degenerates on the set where $|u|$ is infinite. We establish that there exists a relatively open subset $Q_{0}$ of $Q_{T}$ such that $u$ is locally Hölder continuous on $Q_{0}$, the interior of $Q_{T} \backslash Q_{0}$ is empty, and $u \in L^{\infty}\left(Q_{T} \backslash Q_{0}\right)$. Thus $u(x, t)$ may become unbounded only when $(x, t) \in Q_{0}$ approaches the set $Q_{T} \backslash Q_{0}$, the boundary of $Q_{0}$.

Generally speaking, the study of systems of partial differential equations consists of two steps $[\mathrm{K}]$ : first one proves the existence of a solution in a suitable function space; then one proves the regularity of this solution. For some systems, the second step may be false (see [G, Chap. 2]), and the solutions may have singularities. In such cases, one is forced to seek a partial regularity theorem, giving a description of the set of possible singularities. This approach has been used with great success in a variety of settings (see $[\mathrm{K}]$ and the references therein). This paper contains the first partial regularity results for models of the electrical heating of conductors.

Our method relies upon the following weak Harnack inequality:

$$
f_{Q_{2 R}(x, t)} u d y d \tau \leq \operatorname{ess} \inf _{Q_{R}(x, t)} u+c,
$$

where $R>0, Q_{R}(x, t) \equiv\left\{(y, \tau):|y-x|<R\right.$ and $\left.t-R^{2}<\tau \leq t\right\}$, and $c$ is a positive constant. This inequality is a consequence of the following two facts: First, $u$ is a supersolution of the classical heat equation $\frac{\partial u}{\partial t}-\Delta u=0$. Second, $u \in B M O$ $[\mathrm{FS}]$ in the parabolic sense. To be precise, we have

$$
f_{Q_{R}(x, t)}\left(u-u_{R}\right)^{2} d y d \tau \leq c,
$$

where

$$
u_{R}=f_{Q_{R}(x, t)} u d y d \tau
$$

Section 2 is devoted to the proof of (1.4) and (1.5). Our main result is established in Section 3. 
Our system consists of an equation of parabolic type and an equation of elliptic type. Thus, there is no clear theoretical framework available to handle the situation. What saves the game here is a clever combination of some elliptic techniques and some parabolic techniques. Since the second equation does not involve the term $\frac{\partial \varphi}{\partial t}$, we are not able to obtain the Hausdorff dimension of $Q_{T} \backslash Q_{0}$. In this respect, our partial regularity result is different from that in $[\mathrm{G}]$. However, we should be able to remove (1.3). We wish to consider this possibility in a forthcoming paper. For other related work, we refer the reader to $[\mathrm{C}, \mathrm{AC}]$ and references therein.

Finally, we make some remarks about the notation. For $R>0,\left(x_{0}, t_{0}\right) \in \mathbf{R}^{N+1}$, let

$$
\begin{array}{ll}
B_{R}\left(x_{0}\right) & =\left\{x:\left|x-x_{0}\right|<R\right\}, \\
\Omega_{R}\left(x_{0}\right) & =\Omega \cap B_{R}\left(x_{0}\right), \\
Q_{R}\left(x_{0}, t_{0}\right) & =B_{R}\left(x_{0}\right) \times\left(t_{0}-R^{2}, t_{0}\right], \\
D_{R}\left(x_{0}, t_{0}\right) & =\Omega_{R}\left(x_{0}\right) \times\left(t_{0}-R^{2}, t_{0}\right], \\
P_{R}\left(x_{0}, t_{0}\right) & =Q_{R}\left(x_{0}, t_{0}\right) \cap Q_{T}, \\
\partial p P_{R}\left(x_{0}, t_{0}\right) & =\text { the parabolic boundary of } P_{R}\left(x_{0}, t_{0}\right) .
\end{array}
$$

The letter $c$, or $c_{i}, i \in\{0,1, \ldots\}$, is used to denote a generic positive constant. In the expression

we defne

$$
\int_{P_{R}(x, t)}\left(u-u_{R}\right)^{2} d y d \tau
$$

$$
u_{R}=f_{P_{R}(x, t)} u d y d \tau
$$

For $(x, t) \in \bar{Q}_{T}$, define

$$
\begin{aligned}
& R_{*}(x)=\operatorname{dist}(x, \partial \Omega), \\
& R_{0}(x, t)=\min \left\{R_{*}(x), \sqrt{\min \left\{\left(R_{*}(x)\right)^{2}, t\right\}}\right\} .
\end{aligned}
$$

Then

$$
Q_{R_{0}(x, t)}(x, t) \subset Q_{T}
$$

and

$$
Q_{R}(x, t) \cap\left(\mathbf{R}^{N+1} \backslash Q_{T}\right) \neq \emptyset
$$

for each $R>R_{0}(x, t)$.

\section{A WEAK HARNACK INEQUALity FOR $u$}

We first list our assumptions on the data.

(H1) $\sigma \in C(\mathbf{R})$ is such that

$$
0<\sigma \leq M \quad \text { on } \quad \mathbf{R}
$$

for some $M \in(0, \infty)$.

(H2) There exists a function $u^{*}$ in $W^{1, \infty}\left(Q_{T}\right)$ with the properties

$$
\begin{array}{llll}
u^{*}=\bar{u} & \text { on } & S_{T}, \\
u^{*}=u_{0} & \text { on } & \Omega \times\{0\} .
\end{array}
$$

(H3) $\bar{\varphi} \in L^{\infty}\left(0, T ; W^{1, \infty}(\Omega)\right)$. 
We remark that most of our subsequent results require weaker assumptions. For simplicity we will operate under (H1)-(H3) throughout the paper.

In the generality considered here it is not clear to us a priori that $u$ is bounded. As a result, the equation (1.1b) may degenerate. This means that we will not be able to obtain an estimate of the type $|\nabla \varphi| \in L^{p}\left(Q_{T}\right), p \geq 1$. To solve the question of in what sense the system is satisfied, we appeal to the notion of a capacity solution introduced in [X1].

Definition 1. A triplet $\{u, \varphi, g\}$ is said to be a capacity solution of (1.1) if:

(i) $u-u^{*} \in C\left([0, T] ; L^{2}(\Omega)\right) \cap L^{2}\left(0, T ; W_{0}^{1,2}(\Omega)\right), \varphi \in L^{\infty}\left(Q_{T}\right), g \in\left[L^{2}\left(Q_{T}\right)\right]^{N}$;

$$
\begin{gathered}
\frac{\partial u}{\partial t}-\Delta u=\operatorname{div}(\varphi g) \quad \text { in } \quad L^{2}\left(0, T ; W^{-1,2}(\Omega)\right), \\
\operatorname{div} g=0 \quad \text { in } \\
L^{2}\left(0, T ; W^{-1,2}(\Omega)\right) ;
\end{gathered}
$$

(iii) for each $\theta \in C_{0}^{1}(\mathbf{R}), \theta(u) \varphi-\theta\left(u^{*}\right) \bar{\varphi} \in L^{2}\left(0, T ; W_{0}^{1,2}(\Omega)\right)$ and $\theta(u) g=$ $\sigma(u)(\nabla(\theta(u) \varphi)-\varphi \nabla \theta(u))$

(iv) $u(x, 0)=u_{0}(x)$ in $L^{2}(\Omega)$.

A detailed analysis of this definition is presented in [X1]. Here we only point out that (iii) implies $g=\sigma(u) \nabla \varphi$, where $\nabla \varphi$ is a measurable function defined as in $[\mathrm{X} 1]$. A result of $[\mathrm{X} 1]$ asserts that under $(\mathrm{H} 1)-(\mathrm{H} 3)$, problem (1.1) has a capacity solution.

We are ready to state the main result of this section.

Theorem 2. Let $\{u, \varphi\}$ be a capacity solution of (1.1). Then there exists a $c^{*}>0$ depending only on the data such that

$$
f_{Q_{2 R}\left(x_{0}, t_{0}\right)} u(y, \tau) d y d \tau \leq e s s \inf _{Q_{R}\left(x_{0}, t_{0}\right)} u+c^{*}
$$

for all $\left(x_{0}, t_{0}\right) \in Q_{T}, R>0$ such that $2 R \leq R_{0}\left(x_{0}, t_{0}\right)$.

Before we continue, let us cite the following known result.

Lemma 3 (Chain rule). Let $v \in L^{2}\left(0, T ; W_{0}^{1,2}(\Omega)\right)$ be such that

$$
v_{t} \in L^{2}\left(0, T ; W^{-1,2}(\Omega)\right) .
$$

Then for any Lipschitz function $\theta$ with $\theta(0)=0$, the function $t \rightarrow \int_{\Omega}^{u(x, t)} \int_{0}^{\theta} \theta(s) d s d x$ is absolutely continuous on $[0, T]$ and

$$
\frac{d}{d t} \int_{\Omega} \int_{0}^{u(x, t)} \theta(s) d s d x=\left(u_{t}, \theta(u)\right) \quad \text { a.e. } \quad \text { on } \quad(0, T),
$$

where $(\cdot, \cdot)$ denotes the duality pairing between $W^{-1,2}(\Omega)$ and $W_{0}^{1,2}(\Omega)$.

The core of the proof of Theorem 2 is contained in the following lemmas.

Lemma 4. There exists $c>0$ such that

$$
\int_{P_{R}\left(x_{0}, t_{0}\right)} \sigma(u)|\nabla \varphi|^{2} d x d t \leq C R^{N}
$$

for all $R>0$ and $\left(x_{0}, t_{0}\right) \in \bar{Q}_{T}$. 
Proof. First observe from (ii) that

$$
\operatorname{div}(\sigma(u) \nabla \varphi)=0 \quad \text { in } \quad W^{-1,2}(\Omega)
$$

for a.e. $t \in(0, T)$. Now let $\left(x_{0}, t_{0}\right) \in \bar{Q}_{T}, R>0$ be given. Set $B_{R}=B_{R}\left(x_{0}\right)$. Choose $\xi \in C_{0}^{\infty}\left(B_{2 R}\right)$ so that $\xi=1$ on $B_{R}, \xi \geq 0$ on $B_{2 R}$, and $|\nabla \xi| \leq 2 / R$. For each $k \in\{1,2, \ldots\}$, let $\theta_{k} \in C_{0}^{1}(\mathbf{R})$ be such that

$$
\begin{gathered}
\left|\theta_{k}^{\prime}\right| \leq \frac{c}{k}, \\
\lim _{k \rightarrow \infty} \theta_{k}(s)=1 \text { for all } s \in \mathbf{R}, \\
\theta_{k} \geq 0 \quad \text { on } \quad \mathbf{R} .
\end{gathered}
$$

By (iii), $\theta_{k}(u) \varphi-\theta_{k}\left(u^{*}\right) \bar{\varphi} \in W_{0}^{1,2}(\Omega)$ for a.e. $t \in(0, T)$. As a test function in $(2.2)$ we can use $\left(\theta_{k}(u) \varphi-\theta_{k}\left(u^{*}\right) \bar{\varphi}\right) \xi^{2}$ to obtain, for a.e. $t$ in $(0, T)$, that

$$
\begin{aligned}
\int_{\Omega_{2 R}} \sigma(u) & \nabla \varphi \nabla\left(\theta_{k}(u) \varphi\right) \xi^{2} d x \\
= & -\int_{\Omega_{2 R}} \sigma(u) \nabla \varphi \theta_{k}(u) \varphi 2 \xi \nabla \xi d x \\
& +\int_{\Omega_{2 R}} \sigma(u) \nabla \varphi \nabla\left(\theta_{k}\left(u^{*}\right) \bar{\varphi} \xi^{2}\right) d x .
\end{aligned}
$$

In light of (iii), we get, for a.e. $t$ in $(0, T)$, that

$$
\begin{aligned}
\int_{\Omega_{2 R}} \sigma(u)|\nabla \varphi|^{2} \theta_{k}(u) \xi^{2} d x \\
=-\int_{\Omega_{2 R}} \sigma(u) \nabla \varphi \varphi \theta_{k}^{\prime}(u) \nabla u \xi^{2} d x-\int_{\Omega_{2 R}} \sigma(u) \nabla \varphi \theta_{k}(u) \varphi 2 \xi \nabla \xi d x \\
\quad+\int_{\Omega_{2 R}} \sigma(u) \nabla \varphi \nabla\left(\theta_{k}\left(u^{*}\right) \bar{\varphi} \xi^{2}\right) d x .
\end{aligned}
$$

Take $k \rightarrow \infty$, apply Hölder's inequality in the resulting equation, keep in mind (H1)-(H3) and the fact that $\varphi \in L^{\infty}\left(Q_{T}\right) \equiv L^{\infty}\left(0, T ; L^{\infty}(\Omega)\right)$, and thereby obtain

$$
\int_{\Omega_{R}} \sigma(u)|\nabla \varphi|^{2} d x \leq C R^{N-2}
$$

for a.e. $t$ in $(0, T)$. Integration with respect to $t$ yields the desired result.

Lemma 5. There exists $c>0$ such that for all $\left(x_{0}, t_{0}\right) \in Q_{T}$ and $R>0$ with $2 R \leq R_{0}\left(x_{0}, t_{0}\right)$

$$
f_{Q_{2 R}\left(x_{0}, t_{0}\right)} u d x d t \leq \operatorname{ess} \inf _{Q_{R}\left(x_{0}, t_{0}\right)} u+c\left(f_{Q_{2 R}\left(x_{0}, t_{0}\right)}\left(u-u_{2 R}\right)^{2} d x d t\right)^{\frac{1}{2}} .
$$


Proof. Let $\left\{\theta_{k}\right\}$ be given as before. For $\xi \in L^{2}\left(0, T ; W_{0}^{1,2}(\Omega)\right) \cap L^{\infty}\left(Q_{T}\right)$ and $\tau$ in $(0, T)$, we compute, with the aid of (ii) and (iii), that

$$
\begin{aligned}
\int_{0}^{\tau}(\operatorname{div} & \left.(\sigma(u) \nabla \varphi \varphi), \theta_{k}(u) \xi\right) d t \\
= & -\int_{0}^{\tau} \int_{\Omega} \sigma(u) \nabla \varphi \varphi \nabla \theta_{k}(u) \xi d x d t \\
& -\int_{0}^{\tau} \int_{\Omega} \sigma(u) \nabla \varphi \varphi \theta_{k}(u) \nabla \xi d x d t \\
= & -\int_{0}^{\tau} \int_{\Omega} \sigma(u) \nabla \varphi \varphi \nabla \theta_{k}(u) \xi d x d t \\
& -\int_{0}^{\tau} \int_{\Omega} \sigma(u) \nabla \varphi\left(\nabla\left(\xi \varphi \theta_{k}(u)\right)-\xi \nabla\left(\varphi \theta_{k}(u)\right)\right) d x d t \\
= & \int_{0}^{\tau} \int_{\Omega} \sigma(u)|\nabla \varphi|^{2} \theta_{k}(u) \xi d x d t .
\end{aligned}
$$

Letting $k \rightarrow \infty$ yields

$$
\int_{0}^{\tau}(\operatorname{div}(\sigma(u) \varphi \nabla \varphi), \xi) d t=\int_{0}^{\tau} \int_{\Omega} \sigma(u)|\nabla \varphi|^{2} \xi d x d t
$$

for all $\xi \in L^{2}\left(0, T ; W_{0}^{1,2}(\Omega)\right) \cap L^{\infty}\left(Q_{T}\right)$. Now fix $\left(x_{0}, t_{0}\right) \in Q_{T}, 0<2 R \leq$ $R_{0}\left(x_{0}, t_{0}\right)$. Define, for $\varepsilon>0$,

$$
\eta_{\varepsilon}(s)= \begin{cases}1 & \text { if } s \leq 0 \\ -\frac{1}{\varepsilon}(s-\varepsilon) & \text { if } 0<s<\varepsilon \\ 0 & \text { if } s>\varepsilon\end{cases}
$$

Then, for any $A \in \mathbf{R}, \xi \in C_{0}^{\infty}\left(\mathbf{R}^{N+1}\right)$ with $\xi=0$ on $\partial p Q_{T}$, we have $\eta_{\varepsilon}(u-A) \xi \in$ $L^{2}\left(0, T ; W_{0}^{1,2}(\Omega)\right)$. For such $A, \xi$ we deduce from (ii) and (2.4) that for each $\tau \in(0, T]$

$$
\begin{gathered}
\int_{\Omega} \int_{0}^{u-A} \eta_{\varepsilon}(s) d s \xi d x-\int_{0}^{\tau} \int_{\Omega}^{u-A} \int_{0}^{u} \eta_{\varepsilon}(s) d s \xi_{t} d x d t+\int_{0}^{\tau} \int_{\Omega} \nabla u \theta_{\varepsilon}(u-A) \nabla \xi d x d t \\
=-\int_{0}^{\tau} \int_{\Omega}|\nabla u|^{2} \eta_{\varepsilon}^{\prime}(u-A) \xi d x d t+\int_{0}^{\tau} \int_{\Omega} \sigma(u)|\nabla \varphi|^{2} \eta_{\varepsilon}(u-A) \xi d x d t .
\end{gathered}
$$


This implies that $(u-A)^{-}$is a subsolution of the classical heat equation $\frac{\partial u}{\partial t}-\Delta u=$ 0 . That is to say,

$$
\int_{0}^{\tau}\left(\frac{\partial}{\partial t}(u-A)^{-}-\Delta(u-A)^{-}, \xi\right) d t \leq 0
$$

for all $\tau \in(0, T]$ and $\xi \in L^{2}\left(0, T ; W_{0}^{1,2}(\Omega)\right)$ with $\xi=0$ on $\partial p Q_{T}$. This asserts that there exists $c=c(N)>0$ with

$$
\operatorname{ess} \sup _{Q_{R}\left(x_{0}, t_{0}\right)}(u-A)^{-} \leq c\left(f_{Q_{2 R}}\left[(u-A)^{-}\right]^{2} d x d t\right)^{\frac{1}{2}} .
$$

We take $A=f_{Q_{2 R}\left(x_{0}, t_{0}\right)} u d x d t$ and obtain

$$
f_{Q_{2 R}\left(x_{0}, t_{0}\right)} u d x d t \leq \operatorname{ess} \inf _{Q_{R}\left(x_{0}, t_{0}\right)} u+c\left(f_{Q_{2 R}\left(x_{0}, t_{0}\right)}\left[\left(u-u_{2 R}\right)^{-}\right]^{2} d x d t\right)^{\frac{1}{2}} .
$$

This completes the proof.

In view of Lemma 5 , we can obtain Theorem 2 by showing that

$$
f_{Q_{2 R}\left(x_{0}, t_{0}\right)}\left(u-u_{2 R}\right)^{2} d x d t \leq c
$$

for all $\left(x_{0}, t_{0}\right) \in Q_{T}$ and all $0<R$ such that $2 R \leq R_{0}\left(x_{0}, t_{0}\right)$. To this end, set

$$
z(x, t)= \begin{cases}u(x, t)-u^{*}(x, t) & \text { if } \quad(x, t) \in \bar{Q}_{T}, \\ 0 & \text { if } t<0 \text { and } x \in \Omega .\end{cases}
$$

Then one can easily check that $z$ is the weak solution of the following problem

$$
\begin{array}{cc}
\frac{\partial}{\partial t} z-\Delta z=f & \text { in } \quad \Omega \times(-\tau, T], \\
z=0 \quad \text { on } & \partial p(\Omega \times(-\tau, T]),
\end{array}
$$

where $\tau=$ the diameter of $\Omega$ and $f=\operatorname{div}\left(\sigma(u) \varphi \nabla \varphi \chi_{Q_{T}}+\nabla u^{*} \chi_{Q_{T}}\right)-u_{t}^{*} \chi_{Q_{T}}$. Given that $\left(x_{0}, t_{0}\right) \in \bar{Q}_{T} \backslash \Omega \times\{0\}$ and $0<R \leq$ the diameter of $\Omega$, consider the problem

$$
\begin{array}{ccc}
\frac{\partial v}{\partial t}-\Delta v=f \quad \text { in } \quad D_{R} \\
v=0 \quad \text { on } & \partial p D_{R},
\end{array}
$$

where $D_{R}=D_{R}\left(x_{0}, t_{0}\right)$. We can easily conclude from [LSU] that this problem admits a unique solution in

$$
C\left(\left[t_{0}-R^{2}, t_{0}\right] ; L^{2}\left(\Omega_{R}\right)\right) \cap L^{2}\left(\left(t_{0}-R^{2}, t_{0}\right) ; W_{0}^{1,2}\left(\Omega_{R}\right)\right),
$$

where we write $\Omega_{R}$ for $\Omega_{R}\left(x_{0}\right)$. Use $v$ as a test function in (2.7a) to obtain

$$
\begin{aligned}
|v|_{D_{R}} & \equiv \max _{\left[t_{0}-R^{2}, t_{0}\right]} \int_{\Omega_{R}} v^{2}(x, t) d x+\int_{D_{R}}|\nabla v|^{2} d x d t \\
& \leq c \int_{P_{R}} \sigma(u)|\nabla \varphi|^{2} d x d t+c R^{N+2} .
\end{aligned}
$$


For convenience, assume $N \geq 3$. Then an embedding lemma in [D, p.7] indicates that

$$
\|v\|_{2+\frac{4}{N}, D_{R}}^{2} \leq c|v|_{D_{R}} .
$$

Consequently,

$$
\begin{aligned}
\int_{D_{R}} v^{2} d x d t & \leq c R^{2}\left(\int_{D_{R}}|v|^{2+\frac{4}{N}} d x d t\right)^{\frac{N}{N+2}} \\
& \leq c R^{2} \int_{P_{R}} \sigma(u)|\nabla \varphi|^{2} d x d t \\
& \leq c R^{N+2}+C R^{N+4} \leq c_{1} R^{N+2} .
\end{aligned}
$$

The last step is due to Lemma 4.

Let $w=u-v$. Then $w$ is the weak solution of the following problem:

$$
\begin{gathered}
\frac{\partial w}{\partial t}-\Delta w=0 \quad \text { in } \quad D_{R}, \\
w=u \quad \text { in } \quad \partial p D_{R} .
\end{gathered}
$$

Claim 1. If $D_{R} \subset Q_{T}$, i.e., $D_{R}=Q_{R}$, then there exist $c>0, \alpha \in(0,1)$ depending only on $N$ such that

$$
\int_{Q_{\rho}\left(x_{0}, t_{0}\right)}\left(w-w_{\rho}\right)^{2} d x d t \leq c\left(\frac{\rho}{R}\right)^{N+2+2 \alpha} \int_{Q_{R}\left(x_{0}, t_{0}\right)}\left(w-w_{R}\right)^{2} d x d t
$$

for all $0<\rho \leq R$.

We first show that (2.11) holds for $\rho \leq \frac{R}{4}$. To do this, we appeal to a result in [D, p.42] which states that there exist $\alpha \in(0,1)$ and $c>0$, which can be determined a priori only in terms of $N$, such that

$$
\left|w\left(x_{1}, t_{1}\right)-w\left(x_{2}, t_{2}\right)\right| \leq c\left\|w-w_{R}\right\|_{\infty, Q_{\frac{R}{2}}}\left(\frac{\left|x_{1}-x_{2}\right|+\left|t_{1}-t_{2}\right|^{\frac{1}{2}}}{R}\right)^{\alpha}
$$

for all $\left(x_{1}, t_{1}\right),\left(x_{2}, t_{2}\right) \in Q_{\rho}$. Invoking another result in [D, p.123], we obtain that

$$
\left\|w-w_{R}\right\|_{\infty, Q_{\frac{R}{2}}} \leq c(N)\left(f_{Q_{R}}\left(w-w_{R}\right)^{2} d x d t\right)^{\frac{1}{2}} .
$$

Thus, if $0<\rho \leq \frac{R}{4}$, we have that

$$
\begin{aligned}
\int_{Q_{\rho}}\left|w-w_{\rho}\right|^{2} d x d t & \leq c_{1}\left\|w-w_{R}\right\|_{\infty, Q_{\frac{R}{2}}}^{2}\left(\frac{\rho}{R}\right)^{2 \alpha} \cdot \rho^{N+2} \\
& \leq c_{1} f_{Q_{R}}\left(w-w_{R}\right)^{2} d x d t\left(\frac{\rho}{R}\right)^{2 \alpha} \rho^{N+2} \\
& =c_{1}\left(\frac{\rho}{R}\right)^{N+2+2 \alpha} \int_{Q_{R}}\left(w-w_{R}\right)^{2} d x d t .
\end{aligned}
$$


If $\rho>\frac{R}{4}$, then $\frac{4 \rho}{R}>1$. Consequently,

$$
\begin{aligned}
\int_{Q_{\rho}}\left(w-w_{\rho}\right)^{2} d x d t & \leq \int_{Q_{R}}\left(w-w_{R}\right)^{2} d x d t \\
& \leq 4^{2+N+2 \alpha}\left(\frac{\rho}{R}\right)^{2+N+2 \alpha} \int_{Q_{R}}\left(w-w_{R}\right)^{2} d x d t .
\end{aligned}
$$

Then (2.11) holds for $c=\max \left\{c_{1}, 4^{2+N+2 \alpha}\right\}$.

We are ready to prove the following lemma.

Lemma 6. There exist $c_{1}>0, c_{2}>0$ depending only on the data such that for all $\left(x_{0}, t_{0}\right) \in Q_{T}$ and $0<\rho \leq R_{*}=R_{*}\left(x_{0}\right)$,

$$
f_{Q_{\rho}\left(x_{0}, t_{0}\right)}\left(z-z_{\rho}\right)^{2} d x d t \leq c_{1} f_{Q_{R_{*}}\left(x_{0}, t_{0}\right)}\left(z-z_{R_{*}}\right)^{2} d x d t+c_{2}
$$

Proof. Fix $\left(x_{0}, t_{0}\right)$ in $Q_{T}$ and $0<R \leq R_{*}$. Let $v, w$ be given as before. Then for each $0<\rho \leq R$, we calculate, with the aid of (2.9) and (2.11), that

$$
\begin{aligned}
\int_{Q_{\rho}}\left(z-z_{\rho}\right)^{2} d x d t & \\
& \leq c \int_{Q_{\rho}}(z-w)^{2} d x d t+c \int_{Q_{\rho}}\left(w-w_{\rho}\right)^{2} d x d t \\
& \leq c \int_{Q_{R}} v^{2} d x d t+c\left(\frac{\rho}{R}\right)^{N+2+2 \alpha} \int_{Q_{R}}\left(w-w_{R}\right)^{2} d x d t \\
& \leq c\left(1+\left(\frac{\rho}{R}\right)^{N+2+2 \alpha}\right) \int_{Q_{R}} v^{2} d x d t+c\left(\frac{\rho}{R}\right)^{N+2+2 \alpha} \int_{Q_{R}}\left(z-z_{R}\right)^{2} d x d t \\
& \leq c R^{N+2}+c\left(\frac{\rho}{R}\right)^{N+2+2 \alpha} \int_{Q_{R}}\left(z-z_{R}\right)^{2} d x d t .
\end{aligned}
$$

In fact, this holds for all $0<\rho \leq R \leq R_{*}$. Thus, we are in a position to apply Lemma 2.1 in $[\mathrm{G}, \mathrm{p} .86]$. This yields

$$
f_{Q_{\rho}}\left(z-z_{\rho}\right)^{2} d x d t \leq c_{1} f_{Q_{R}}\left(z-z_{R}\right)^{2} d x d t+c_{2}
$$

for all $0 \leq \rho \leq R \leq R_{*}$. Set $R=R_{*}$ to obtain the desired result.

If $\left(x_{0}, t_{0}\right) \in Q_{T}$ is such that $R_{0}\left(x_{0}, t_{0}\right) \leq R_{*}\left(x_{0}\right)$, we have, for all $0<\rho \leq$ $R_{0}\left(x_{0}, t_{0}\right)$, that

$$
\begin{aligned}
f_{Q_{\rho}}\left(u-u_{\rho}\right)^{2} d x d t & \leq c f_{Q_{\rho}}\left(z-z_{\rho}\right)^{2}+c f_{Q_{\rho}}\left(u^{*}-\left(u^{*}\right)_{\rho}\right)^{2} d x d t \\
& \leq c f_{Q_{R_{*}}}\left(z-z_{\rho}\right)^{2} d x+c \\
& \leq c f_{Q_{R^{*}}} z^{2} d x+c .
\end{aligned}
$$


This implies that for all $(x, t) \in Q_{T}$ such that dist $(x, \partial \Omega) \geq d>0$, there exists a $c=c(d)$ with

$$
\begin{aligned}
f_{Q_{\rho}(x, t)}\left(u-u_{\rho}\right)^{2} d x d t & \leq c f_{Q_{R_{0}}(x, t)}\left(u-u_{R_{0}}\right)^{2} d x d t+c \\
& \leq c(d)
\end{aligned}
$$

for all $0<\rho \leq R_{0}(x, t)$. To finish the proof of (2.5), we still have to show that for each $\left(x_{0}, t_{0}\right) \in S_{T}$, there is a $\rho_{0}>0$ such that $f_{Q_{R_{0}}(x, t)}\left(u-u_{R_{0}}\right)^{2} d y d \tau$ is bounded in $D_{\rho_{0}}\left(x_{0}, t_{0}\right)$. The idea here is to introduce a change of variables which flattens the relevant portion of the boundary $\partial \Omega$. Fix $x_{0} \in \partial \Omega$. Since $\Omega$ is $C^{1}$, we can find an open neighborhood $U$ about $x_{0}$, a number $A_{0}>0$, a $C^{1}$-homeomorphism $\mathcal{T}$ between $\Lambda \equiv\left\{y \in \mathbf{R}^{N}:\left|y_{i}\right|<A_{0}, i=1, \ldots, N\right\}$ and $U$ such that

$$
\begin{array}{ll}
\mathcal{T}^{-1}(U \cap \Omega) & =\Lambda^{+} \equiv\left\{y \in \Lambda: 0<y_{N}\right\}, \\
\mathcal{T}^{-1}\left(U \cap\left(\mathbf{R}^{N} \backslash \bar{\Omega}\right)\right) & =\Lambda^{-} \equiv\left\{y \in \Lambda: y_{N}<0\right\}, \\
\mathcal{T}^{-1}\left(x_{0}\right) & =0, \\
\mathcal{T}^{-1}(U \cap \partial \Omega) & =\left\{y \in \Lambda: y_{N}=0\right\} .
\end{array}
$$

Let $\mathcal{T}$ be defined by

$$
x_{i}=g_{i}\left(y_{1}, \ldots, y_{N}\right), \quad i=1, \ldots, N
$$

Then set

$$
H=\left(\frac{\partial}{\partial y_{j}} g_{i}\right)
$$

Following the construction of $\mathcal{T}$ in $[\mathrm{SW}]$, we can choose $g_{i}$ so that

$$
\text { the Jacobian of } \mathcal{T} \equiv|\operatorname{det} H|=1 \text {. }
$$

For $y \in \Lambda^{+}$, define

$$
\widetilde{z}(y, t)=z\left(g_{1}\left(y_{1}, \ldots y_{N}\right), \cdots, g_{N}\left(y_{1}, \ldots, y_{N}\right), t\right) .
$$

An elementary calculation shows

$$
\frac{\partial}{\partial t} \widetilde{z}-\operatorname{div}\left(\left(H^{-1}\right)^{T} H^{-1} \nabla \widetilde{z}\right)=\operatorname{div} g+g_{0} \quad \text { in } \quad \Lambda^{+} \times(-\tau, T],
$$

where

$$
\begin{array}{ll}
g(y, t) & =\left.H^{-1}\left(\sigma(u) \varphi \nabla \varphi \chi_{Q_{T}}+\nabla u^{*} \chi_{Q_{T}}\right)\right|_{x=\mathcal{T} y}, \\
g_{0}(y, t) & =-\left.u_{t}^{*} \chi_{Q_{T}}\right|_{x=\mathcal{T} y}, \\
\left(H^{-1}\right)^{T} H^{-1} & =\left.\left(H^{-1}\right)^{T} H^{-1}\right|_{x=\mathcal{T} y} .
\end{array}
$$

Note that in the derivation of (2.17) we made use of (2.16). Now set

$$
\bar{z}(y, t)=\left\{\begin{array}{ccc}
\widetilde{z}(y, t) & \text { if } & y_{N}>0, \\
-\widetilde{z}\left(y_{1}, \ldots y_{N-1},-y_{N}, t\right) & \text { if } & y_{N}<0 .
\end{array}\right.
$$

Then $\bar{z}$ satisfies

$$
\frac{\partial}{\partial t} \bar{z}-\operatorname{div}(\bar{H} \nabla \bar{z})=\operatorname{div} \bar{g}+\bar{g}_{0} \quad \text { in } \quad \Lambda \times(-\tau, T],
$$


where

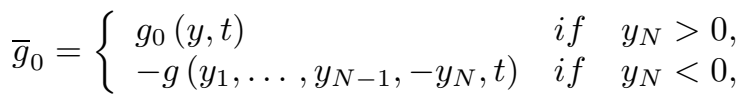

$$
\begin{aligned}
& \bar{H}=\left\{\begin{array}{ccc}
\left(H^{-1}\right)^{T} H^{-1} & \text { if } & y_{N}>0, \\
\bar{I}\left(\left(H^{-1}\right)^{T} H^{-1}\right)\left(y_{1}, \ldots, y_{N-1},-y_{N}\right) \bar{I} & \text { if } & y_{N}<0,
\end{array}\right. \\
& \bar{I}=\left(\begin{array}{ccccc}
1 & 0 & \cdots & 0 & 0 \\
0 & 1 & \cdots & 0 & 0 \\
0 & 0 & \cdots & 1 & 0 \\
0 & 0 & \cdots & 0 & -1
\end{array}\right) \\
& \bar{g}=\left\{\begin{array}{ccc}
g & \text { if } & y_{N}>0, \\
-\bar{I} g\left(y_{1}, \ldots, y_{N-1},-y_{N}, t\right) & \text { if } & y_{N}<0 .
\end{array}\right.
\end{aligned}
$$

Observe that $\bar{H}$ is still positive definite. In fact, in view of (4.6) in [SW], we can choose $g_{i}$ so that $\bar{H}$ is continuous in $\Lambda$. We are ready to conclude from our earlier argument that

$$
f_{Q_{\rho}\left(y_{0}, t_{0}\right)}\left(\bar{z}-(\bar{z})_{\rho}\right)^{2} d y d t \leq c\left(A_{0}\right)
$$

for all $\left(y_{0}, t_{0}\right)$ such that $\left|y_{0}\right| \leq \frac{A_{0}}{2}, t_{0} \in(0, T]$, and $0<\rho \leq \frac{A_{0}}{2}$. It is easy to see that

$$
\begin{gathered}
\text { meas } \mathcal{T}\left(B_{\rho}\left(y_{0}\right)\right)=\text { meas } B_{\rho}\left(y_{0}\right), \\
B_{2 \rho}\left(\mathcal{T}^{-1}\left(x_{1}\right)\right) \supset \mathcal{T}^{-1}\left(B_{\rho}\left(x_{1}\right)\right)
\end{gathered}
$$

for all $x_{1} \in U, \rho>0$ with $B_{\rho}\left(x_{1}\right) \subset U$. For $\left(x_{1}, t_{1}\right) \in \mathcal{T}\left(\Lambda^{+} \cap B_{\frac{A_{0}}{2}}(0)\right) \times(0, T]$ such that $2 R_{0}\left(x_{1}, t_{1}\right) \leq \frac{A_{0}}{2}$, we compute, with the aid of (2.19), (2.20), and (2.21), that

$$
\begin{aligned}
f_{Q_{R_{0}}\left(x_{1}, t_{1}\right)}\left(z-z_{\rho}\right)^{2} d x d t & =c \frac{1}{R_{0}^{N+2}} \int_{t_{1}-R_{0}^{2}}^{t_{1}} \int_{\mathcal{T}^{-1}\left(B_{R_{0}}\left(x_{1}\right)\right)}\left(\widetilde{z}-(\widetilde{z})_{\rho}\right)^{2} d y d t \\
& \leq c f_{Q_{2 R_{0}}\left(\mathcal{T}-1\left(x_{1}\right), t_{1}\right)}\left(\bar{z}-(\bar{z})_{2 R_{0}}\right)^{2} d y d t \\
& \leq c .
\end{aligned}
$$

This completes the proof of (2.5). Hence Theorem 2 follows.

Theorem 7. Let $\sigma, u^{*}, \bar{\varphi}$ be given as before. Then any bounded capacity solution $(u, \varphi)$ to (1.1) satisfies

(P1) $u \in C^{\alpha, \frac{\alpha}{2}}\left(\bar{Q}_{T}\right)$ for some $\alpha \in(0,1)$;

(P2) for each $q>1,|\nabla u| \in L^{q}\left(0, T ; W^{1, q}(\Omega)\right)$ and $|\nabla \varphi| \in L^{\infty}\left(0, T ; W^{1, q}(\Omega)\right)$.

Since $u$ is bounded, $\sigma(u)$ stays away from 0 below. Thus, this result is essentially the same as that in [YL]. The proof there is based upon an application of the single-layer potential theory. We claim that this result is a consequence of our 
earlier argument. To see this, note that for a.e. $t$ in $(0, T), \varphi(x, t)$ solves the boundary-value problem

$$
\begin{gathered}
\operatorname{div}(\sigma(u) \nabla \varphi)=0 \quad \text { in } \quad \Omega, \\
\varphi=\bar{\varphi} \quad \text { on } \quad \partial \Omega .
\end{gathered}
$$

Hence, the classical regularity theory for linear elliptic equations asserts

$$
\text { ess } \sup _{(0, T)}\|\varphi(\cdot, t)\|_{C^{\beta}(\bar{\Omega})} \leq c
$$

for some $\beta \in(0,1)$. Set $\varphi_{R}(t)=f_{\Omega_{R}} \varphi(y, t) d y$. Then we have

$$
\left\|\varphi-\varphi_{R}\right\|_{\infty, P_{R}} \leq c R^{\beta} \text {. }
$$

Observe from (2.22a) that

$$
\operatorname{div}(\sigma(u) \varphi \nabla \varphi)=\operatorname{div}\left(\sigma(u)\left(\varphi-\varphi_{R}\right) \nabla \varphi\right) .
$$

Thus, $f$ in (2.7a) may be replaced by

$$
f=\operatorname{div}\left(\sigma(u)\left(\varphi-\varphi_{R}\right) \nabla \varphi \chi_{Q_{T}}+\nabla u^{*} \chi_{Q_{T}}\right)-u_{t}^{*} \chi_{Q_{T}}
$$

and $|v|_{D_{R}}$ in (2.8) may be estimated by

$$
\begin{aligned}
|v|_{D_{R}} & \leq c \int_{P_{R}} \sigma(u)\left(\varphi-\varphi_{R}\right)^{2}|\nabla \varphi|^{2} d x d t+C R^{N+2} \\
& \leq C R^{N+2 \beta}+c R^{N+2}
\end{aligned}
$$

Consequently,

$$
\int_{D_{R}} v^{2} d x d t \leq c R^{N+2+2 \beta}
$$

So (2.13) now reads

$$
\int_{Q_{\rho}}\left(z-z_{\rho}\right)^{2} d x d t \leq c R^{N+2+2 \beta}+c\left(\frac{\rho}{R}\right)^{N+2+2 \alpha} \int_{Q_{R}}\left(z-z_{R}\right)^{2} d x d t
$$

for all $0<\rho \leq R \leq R_{*}$. Pick $\gamma$ from $(0, \min \{\alpha, \beta\})$. Then we deduce from [G, p.86] that

$$
\int_{Q_{\rho}}\left(z-z_{\rho}\right)^{2} d x d t \leq c \rho^{N+2+2 \gamma}+c\left(\frac{\rho}{R_{*}}\right)^{N+2+2 \gamma} \int_{Q_{R_{*}}}\left(z-z_{R_{*}}\right)^{2} d x d t
$$

for all $0<\rho \leq R_{*}$. By chasing the proof in [G,pp.70-72], we obtain that $z$ is locally Hölder continuous in $\bar{Q}_{T} \backslash \bar{S}_{T}$. The local Hölder continuity at the lateral boundary $S_{T}$ can be obtained from (2.18). Once we know $\sigma(u)$ is continuous, a result in [R, p.82] states that $|\nabla \varphi| \in L^{q}(\Omega)$ for each $q>1$ and a.e. $t$ in $(0, T)$. In fact, we have

$$
\text { ess } \sup _{(0, T)}\|\nabla \varphi\|_{q, \Omega} \leq c .
$$

This, in turn, implies

$$
|\nabla u| \in L^{q}\left(0, T ; W^{1, q}(\Omega)\right)
$$

for each $q>1$, because of a result in [BLP, pp. 273-274]. 


\section{Partial REgUlarity FOR $u$}

The main result of this section is:

Theorem 8. Assume (H1)-(H3), (1.2), and (1.3). Let $(u, \varphi)$ be the solution constructed in [X2]. Then there exists a relatively open subset $Q_{0}$ of $Q_{T}$ such that

a. $u$ is locally Hölder continuous on $Q_{0}$;

b. $Q_{T} \backslash Q_{0}$ has an empty interior;

c. $u \in L^{\infty}\left(Q_{T} \backslash Q_{0}\right)$.

The solution in [X2] is established as a limit of a sequence of approximate solutions. As we shall see, our proof of Theorem 8 relies on the approximation procedure employed in [X2]. Since classical weak solutions or capacity solutions are not known to be unique, we do not know if Theorem 8 holds for a solution obtained by other constructions. Note that the results in Section 2 hold for any capacity solutions.

We are not able to obtain the Hausdorff dimension of $Q_{T} \backslash Q_{0}$ due to the degeneracy involved.

Now consider a sequence of approximate problems:

$$
\begin{array}{r}
\frac{\partial u_{n}}{\partial t}-\Delta u_{n}=\sigma_{n}\left(u_{n}\right)\left|\nabla \varphi_{n}\right|^{2} \quad \text { in } \quad Q_{T}, \\
\operatorname{div}\left(\sigma_{n}\left(u_{n}\right) \nabla \varphi_{n}\right)=0 \quad \text { in } \quad Q_{T}, \\
u_{n}=u^{*} \quad \text { on } \quad \partial p Q_{T}, \\
\varphi_{n}=\bar{\varphi} \quad \text { on } \quad S_{T} \quad(n=1,2, \ldots),
\end{array}
$$

where $\sigma_{n}(s)=\sigma(s)+\frac{1}{n}$. Then by virtue of a result in [X2] $(u, \varphi)$ in Theorem 8 can be viewed as a strong limit of $\left(u_{n}, \varphi_{n}\right)$ in $\left[L^{2}\left(Q_{T}\right)\right]^{2}$. For each fixed $n, u_{n}$ satisfies the conclusions of Theorem 7 . We shall proceed to derive a priori estimates for $\left(u_{n}, \varphi_{n}\right)$.

Lemma 9. There exists a positive constant $c$ such that

$$
\begin{gathered}
\left\|\varphi_{n}\right\|_{\infty, Q_{T}} \leq c, \\
\int_{Q_{T}} \sigma_{n}\left(u_{n}\right)\left|\nabla \varphi_{n}\right|^{2} d x d t \leq c, \\
\operatorname{ess} \sup _{[0, T]} \int_{\Omega} u_{n}^{2}(x, t) d x+\int_{Q_{T}}\left|\nabla u_{n}\right|^{2} d x d t \leq c
\end{gathered}
$$

for all $n$.

We refer the reader to [X2] for the proof.

Lemma 10. For each $A>0$,

$$
-\Delta \varphi_{n}=\operatorname{div}\left(F_{A}\left(u_{n}\right) \nabla \varphi_{n}\right)+G_{A}\left(u_{n}\right) \nabla \varphi_{n} \nabla u_{n} \quad \text { in } \quad \mathcal{D}^{\prime}(\Omega)
$$

for a.e. $t$ in $(0, T)$, where

$$
\begin{gathered}
F_{A}\left(u_{n}\right)=\sigma_{n}\left(u_{n}\right) \frac{1}{A} \int_{u_{n}}^{u_{n}+A} \frac{1}{\sigma_{n}(s)} d s-1, \\
G_{A}\left(u_{n}\right)=\frac{1}{A}\left(1-\frac{\sigma_{n}\left(u_{n}\right)}{\sigma_{n}\left(u_{n}+A\right)}\right) .
\end{gathered}
$$


Proof. For each $A>0$ and each $\xi \in C_{0}^{\infty}(\Omega)$, we have that

$$
\xi \int_{u_{n}}^{u_{n}+A} \frac{1}{\sigma_{n}(s)} d s \in W_{0}^{1,2}(\Omega)
$$

for each $t$ in $[0, T]$. Observe from $(3.1 \mathrm{~b})$ that

$$
\operatorname{div}\left(\sigma_{n}\left(u_{n}\right) \nabla \varphi_{n}\right)=0 \quad \text { in } \quad \mathcal{D}^{\prime}(\Omega)
$$

for a.e. $t$ in $(0, T)$. We conclude that for a.e. $t$ in $(0, T)$

$$
\begin{aligned}
& \int_{\Omega} \sigma_{n}\left(u_{n}\right) \nabla \varphi_{n} \int_{u_{n}}^{u_{n}+A} \frac{1}{\sigma_{n}(s)} d s \nabla \xi d x \\
& \quad+\int_{\Omega} \sigma_{n}\left(u_{n}\right) \nabla \varphi_{n} \xi\left(\frac{1}{\sigma_{n}\left(u_{n}+A\right)}-\frac{1}{\sigma_{n}\left(u_{n}\right)}\right) \nabla u_{n} d x=0 .
\end{aligned}
$$

This yields the lemma.

From now on, we will operate under (1.2) and (1.3). It is easy to see that

$$
\left|\frac{\sigma_{n}(s+\tau)}{\sigma_{n}(s)}-1\right| \leq\left|\frac{\sigma(s+\tau)}{\sigma(s)}-1\right|
$$

A lemma in [X2] indicates that if (1.2) holds, then for each $L>0$ there exist $m_{L}>0, M_{L}>0$ with the property

$$
m_{L} \leq \frac{\sigma(s+\tau)}{\sigma(s)} \leq M_{L} \quad \text { for } \quad(s, \tau) \in(-\infty, \infty) \times[-L, L] .
$$

If this holds, then

$$
m_{L} \leq \frac{\sigma_{n}(s+\tau)}{\sigma_{n}(s)} \leq M_{L} \quad \text { for } \quad(s, \tau) \in(-\infty, \infty) \times[-L, L] .
$$

In this case one can show [X2] that $\int_{Q_{T}}\left|\nabla \varphi_{n}\right|^{2} d x d t \leq c$ for all $n$. Thus $(u, \varphi)$ is a classical weak solution.

Theorem 11. For each $q>2$ there exists $c>0$ such that

$$
\left\|\nabla \varphi_{n}\right\|_{q, Q_{\rho}(x, t)} \leq c\left(\left\|\nabla u_{n}\right\|_{q, Q_{R}(x, t)}+\frac{R^{\frac{N+2}{q}}}{R-\rho}\right)
$$

for all $(x, t) \in Q_{T}$ and $0<\rho<R \leq R_{0}(x, t)$.

Proof. Let $x_{0} \in \Omega$ be given. Fix $R_{*}\left(x_{0}\right)>R>r>0$. Choose $\xi \in C_{0}^{\infty}\left(B_{R}\left(x_{0}\right)\right)$ so that

$$
\begin{gathered}
\xi \geq 0 \quad \text { on } \quad B_{R} \equiv B_{R}\left(x_{0}\right), \\
\xi=1 \quad \text { on } \quad B_{r},
\end{gathered}
$$

and

$$
|\nabla \xi| \leq \frac{c}{R-r}
$$

Set

$$
\psi_{n}=\varphi_{n} \xi
$$


A simple calculation yields

$$
\begin{aligned}
-\Delta \psi_{n}= & \operatorname{div}\left(F_{A}\left(u_{n}\right) \nabla \psi_{n}\right)-\left(1+F_{A}\left(u_{n}\right)\right) \nabla \varphi_{n} \nabla \xi \\
& +G_{A}\left(u_{n}\right) \nabla \psi_{n} \nabla u_{n}-G_{A}\left(u_{n}\right) \nabla \xi \varphi_{n} \nabla u_{n} \\
& -\operatorname{div}\left(\left(1+F_{A}\left(u_{n}\right)\right) \varphi_{n} \nabla \xi\right) \quad \text { in } \quad \mathbf{R}^{N}
\end{aligned}
$$

for a.e. $t$ in $(0, T)$, where $\varphi_{n}, u_{n}$ are understood to be zero outside $B_{R}$. Now for each $y \in \mathbf{R}^{N}$ and $\rho_{0}>0$ consider the problem

$$
\begin{array}{ccc}
-\Delta \phi_{n}=0 \quad \text { in } & B_{\rho_{0}}(y) \equiv B_{\rho_{0}}, \\
\phi_{n}=\psi_{n} \quad \text { on } & \partial B_{\rho_{0}} .
\end{array}
$$

A result in $[\mathrm{G}, \mathrm{p} .78]$ asserts that

$$
\int_{B_{\rho}}\left|\nabla \phi_{n}-\left(\nabla \phi_{n}\right)_{\rho}\right|^{2} d x \leq c\left(\frac{\rho}{\rho_{0}}\right)^{N+2} \int_{B_{\rho_{0}}}\left|\nabla \phi_{n}-\left(\nabla \phi_{n}\right)_{\rho_{0}}\right|^{2} d x
$$

for all $0<\rho \leq \rho_{0}$, where $c>0$ depends only on $N$. Subtract (3.12a) from (3.11), use $\psi_{n}-\phi_{n}$ as a test function in the resulting equation, and thereby obtain

$$
\begin{aligned}
\int_{B_{\rho_{0}}} \mid \nabla & \left.\left(\psi_{n}-\phi_{n}\right)\right|^{2} d x \\
\leq & \int_{B_{\rho_{0}}} F_{A}^{2}\left(u_{n}\right)\left|\nabla \psi_{n}\right|^{2} d x+c \int_{B_{\rho_{0}}}\left(1+F_{A}\left(u_{n}\right)\right)\left|\nabla \varphi_{n}\right||\nabla \xi| d x \\
& +c \int_{B_{\rho_{0}}} G_{A}\left(u_{n}\right)\left|\nabla \psi_{n}\right|\left|\nabla u_{n}\right| d x+c \int_{B_{\rho_{0}}} G_{A}\left(u_{n}\right)\left|\nabla \xi \nabla u_{n}\right| d x \\
& +c \int_{B_{\rho_{0}}}|\nabla \xi|^{2}\left(1+F_{A}\left(u_{n}\right)\right)^{2} d x .
\end{aligned}
$$

Here, we used the fact that $\left\{\phi_{n}\right\},\left\{\psi_{n}\right\},\left\{\varphi_{n}\right\}$ are all bounded in $L^{\infty}\left(Q_{T}\right)$. Observe that there exists a $\theta_{n}$ in $(0,1)$ so that

$$
\frac{1}{A} \int_{u_{n}}^{u_{n}+A} \frac{1}{\sigma_{n}(s)} d s=\frac{1}{\sigma_{n}\left(u_{n}+\theta_{n} A\right)}
$$

We can infer from (3.9) that

$$
\left|1+F_{A}\left(u_{n}\right)\right| \leq c(A),\left|G_{A}\left(u_{n}\right)\right| \leq c(A)
$$


for some $c(A)>0$. For any $\varepsilon>0$, we conclude from (3.14) that

$$
\begin{aligned}
\int_{B_{\rho_{0}}} \mid \nabla & \left.\left(\psi_{n}-\phi_{n}\right)\right|^{2} d x \\
\leq & \left(\left\|F_{A}^{2}\left(u_{n}\right)\right\|_{\infty, B_{R}}+\varepsilon\right) M\left(\left|\nabla \psi_{n}\right|^{2}\right) \rho_{0}^{N} \\
& +\rho_{0}^{N}\left(\frac{c(A)}{(R-r)} M\left(\left|\nabla \varphi_{n}\right|\right)+c(\varepsilon, A) M\left(\left|\nabla u_{n}\right|^{2}\right)+c(A) M\left(|\nabla \xi|^{2}\right)\right) \\
\equiv & D_{n} \rho_{0}^{N}+E_{n} \rho_{0}^{N},
\end{aligned}
$$

where $M\left(\left|\nabla \psi_{n}\right|^{2}\right), M\left(\left|\nabla u_{n}\right|^{2}\right), M\left(\left|\nabla \varphi_{n}\right|\right)$, and $M\left(|\nabla \xi|^{2}\right)$ are the maximal functions of $\left|\nabla \psi_{n}\right|^{2},\left|\nabla u_{n}\right|^{2} \chi_{B_{R}},\left|\nabla \varphi_{n}\right| \chi_{B_{R}}$, and $|\nabla \xi|^{2}$, respectively. For $0<\rho \leq \rho_{0}$, we estimate, with the aid of (3.13) and (3.15), that

$$
\begin{gathered}
\int_{B_{\rho}}\left(\nabla \psi_{n}-\left(\nabla \psi_{n}\right)_{\rho}\right)^{2} d x \\
\leq c \int_{B_{\rho}}\left(\nabla \psi_{n}-\nabla \phi_{n}\right)^{2} d x+\int_{B_{\rho}}\left(\nabla \phi_{n}-\left(\nabla \phi_{n}\right)_{\rho}\right)^{2} d x \\
\leq c \int_{B_{\rho_{0}}}\left(\nabla \psi_{n}-\nabla \phi_{n}\right)^{2} d x+c\left(\frac{\rho}{\rho_{0}}\right)^{N+2} \int_{B_{\rho_{0}}}\left(\nabla \psi_{n}-\left(\nabla \psi_{n}\right)_{\rho_{0}}\right)^{2} d x \\
\leq c\left(D_{n}+E_{n}\right) \rho_{0}^{N}+c\left(\frac{\rho}{\rho_{0}}\right)^{N+2} \int_{B_{\rho_{0}}}\left(\nabla \psi_{n}-\left(\nabla \psi_{n}\right)_{\rho_{0}}\right)^{2} d x \\
\leq c\left(D_{n}+E_{n}\right) \rho_{0}^{N} \\
\quad+c\left(\frac{\rho}{\rho_{0}}\right)^{N+2}\left|\nabla \psi_{n}\right|^{2} d x .
\end{gathered}
$$

We are ready to employ an argument in [DM]. For this purpose, we rewrite (3.16) as

$$
\begin{aligned}
& f_{B_{\rho}}\left(\nabla \psi_{n}-\left(\nabla \psi_{n}\right)_{\rho}\right)^{2} d x \\
& \quad \leq c\left[\left(\left\|F_{A}^{2}\left(u_{n}\right)\right\|_{\infty, B_{R}}+\varepsilon\right)\left(\frac{\rho_{0}}{\rho}\right)^{N}+\left(\frac{\rho}{\rho_{0}}\right)^{2}\right] M\left(\left|\nabla \psi_{n}\right|^{2}\right)+c\left(\frac{\rho_{0}}{\rho}\right)^{N} E_{n}
\end{aligned}
$$

Remember that

$$
\left|F_{A}\left(u_{n}\right)\right| \leq\left|\frac{\sigma\left(u_{n}\right)}{\sigma\left(u_{n}+\theta_{n} A\right)}-1\right| \rightarrow 0
$$

uniformly as $A \rightarrow 0$. Thus, for each $\tau \in(0,1)$, we may choose $A$ and $\varepsilon$ so that

$$
f_{B_{\rho_{0} \tau}}\left(\nabla \psi_{n}-\left(\nabla \psi_{n}\right)_{\rho_{0} \tau}\right)^{2} d x \leq c \tau^{2} M\left(\left|\nabla \psi_{n}\right|^{2}\right)+c(\tau) E_{n} .
$$

Since $\rho_{0}$ is arbitrary, we can conclude that

$$
\left(\left(\nabla \psi_{n}\right)^{\sharp}\right)^{2} \leq c \tau^{2} M\left(\left|\nabla \psi_{n}\right|^{2}\right)+c(\tau) E_{n},
$$


where $\left(\nabla \psi_{n}\right)^{\sharp}$ is the sharp maximal function associated with $\nabla \psi_{n}$. Remember that $u_{n} \in W^{1, q}(\Omega)$ for a.e. $t$ in $(0, T)$ and each $q>2$. Now we are in a position to apply the Fefferman-Stein inequality in [FS]. This results in

$$
\begin{aligned}
\int_{B_{R}}\left|\nabla \psi_{n}\right|^{q} d x & =\int_{\mathbf{R}^{N}}\left|\nabla \psi_{n}\right|^{q} d x \\
& \leq \int_{\mathbf{R}^{N}}\left(M\left(\nabla \psi_{n}\right)\right)^{q} d x \\
& \leq c(q) \int_{\mathbf{R}^{N}}\left[\left(\nabla \psi_{n}\right)^{\sharp}\right]^{q} d x \\
& \leq c(q) \tau^{q} \int_{\mathbf{R}^{N}}\left(M\left(\left|\nabla \psi_{n}\right|^{2}\right)\right)^{\frac{q}{2}} d x+c(\tau, q) E_{n}^{\frac{q}{2}}
\end{aligned}
$$

for each $q>2$. Recalling the definition of $E_{n}$ and the Hardy-Littlewood maximal theorem $[\mathrm{DM}]$, we arrive at

$$
\begin{aligned}
\int_{\mathbf{R}^{N}}\left|\nabla \psi_{n}\right|^{q} d x \leq & c(q) \tau^{q} \int_{\mathbf{R}^{N}}\left|\nabla \psi_{n}\right|^{q} d x+\frac{c(q)}{(R-r)^{\frac{q}{2}}} \int_{B_{R}}\left|\nabla \varphi_{n}\right|^{\frac{q}{2}} d x \\
& +c(q) \int_{B_{R}}\left|\nabla u_{n}\right|^{q} d x+c(q) \int_{\mathbf{R}^{N}}|\nabla \xi|^{q} d x .
\end{aligned}
$$

Now choose $\tau$ so that $c(q) \tau^{q}<\frac{1}{2}$. We derive from (3.18) that

$$
\begin{aligned}
\int_{B_{r}}\left|\nabla \varphi_{n}\right|^{q} d x \leq \int_{\mathbf{R}^{N}}\left|\nabla \psi_{n}\right|^{q} d x \\
\quad \leq \frac{c}{(R-r)^{\frac{q}{2}}} \int_{B_{R}}\left|\nabla \varphi_{n}\right|^{\frac{q}{2}} d x+c \int_{B_{R}}\left|\nabla u_{n}\right|^{q} d x+c \frac{R^{N}}{(R-r)^{q}} \\
\quad \leq \varepsilon \int_{B_{R}}\left|\nabla \varphi_{n}\right|^{q} d x+c \int_{B_{R}}\left|\nabla u_{n}\right|^{q} d x+\frac{c(\varepsilon) R^{N}}{(R-r)^{q}}
\end{aligned}
$$

for each $\varepsilon>0$. For $k \in\{1,2, \ldots\}$, define

$$
\rho_{k}=R-\frac{\theta R}{2^{k}}
$$

where $\theta \in(0,1)$. Set $r=\rho_{k}, R=\rho_{k+1}$ in (3.19) to obtain

$$
\begin{aligned}
\int_{B_{\rho_{k}}}\left|\nabla \varphi_{n}\right|^{q} d x \leq & \varepsilon \int_{B_{\rho_{k+1}}}\left|\nabla \varphi_{n}\right|^{q} d x+c \int_{B_{R}}\left|\nabla u_{n}\right|^{q} d x \\
& +\frac{c(\varepsilon) R^{N-q} 2^{q k}}{\theta^{q}} .
\end{aligned}
$$


In view of a result in $[\mathrm{D}, \mathrm{p} .13]$, we derive, by suitably selecting $\varepsilon$, that

$$
\int_{R(1-\theta)}\left|\nabla \varphi_{n}\right|^{q} d x \leq c(q) \int_{B_{R}}\left|\nabla u_{n}\right|^{q} d x+c(q) \frac{R^{N}}{(R \theta)^{q}} .
$$

A simple integration yields (3.10).

Proof of Theorem 8. Fix $\left(x_{0}, t_{0}\right)$ in $Q_{T}$. Let $R>0$ be such that $Q_{R}\left(x_{0}, t_{0}\right) \equiv$ $Q_{R} \subset Q_{T}$. Consider the problem

$$
\begin{gathered}
\frac{\partial v_{n}}{\partial t}-\Delta v_{n}=\operatorname{div}\left(\sigma_{n}\left(u_{n}\right) \varphi_{n} \nabla \varphi_{n}\right) \quad \text { in } \quad Q_{R}, \\
v_{n}=0 \quad \text { on } \quad \partial p Q_{R} .
\end{gathered}
$$

In light of a result in [BLP, pp.273-274], we have that for each $q>2$ there exists a $c(q)$ independent of $R$ such that

$$
\left\|\nabla v_{n}\right\|_{q, Q_{R}} \leq c(q)\left\|\sigma_{n}\left(u_{n}\right) \varphi_{n} \nabla \varphi_{n}\right\|_{q, Q_{R}} .
$$

Set $w_{n}=u_{n}-v_{n}$. Then $w_{n}$ satisfies

$$
\begin{gathered}
\frac{\partial w_{n}}{\partial t}-\Delta w_{n}=0 \quad \text { in } \quad Q_{R} \\
w_{n}=u_{n} \quad \text { on } \quad \partial p Q_{R} .
\end{gathered}
$$

We infer from a result in $[D]$ that

$$
\left\|\nabla w_{n}\right\|_{\infty, Q_{\rho}} \leq \frac{c}{(R-\rho)^{\frac{N+2}{2}}}\left(\int_{Q_{R}}\left|\nabla w_{n}\right|^{2} d x d t\right)^{\frac{1}{2}}
$$

for each $0<\rho<R$. Keeping these in mind, we calculate, for $\rho<R$, that

$$
\begin{aligned}
\left\|\nabla u_{n}\right\|_{q, Q_{\rho}} \leq & \left\|\nabla v_{n}\right\|_{q, Q_{R}}+\left\|\nabla w_{n}\right\|_{q, Q_{\rho}} \\
\leq & \left\|\nabla v_{n}\right\|_{q, Q_{R}}+\rho^{\frac{N+2}{q}}\left\|\nabla w_{n}\right\|_{\infty, Q_{\rho}} \\
\leq & c(q)\left\|\sigma_{n}\left(u_{n}\right) \varphi_{n} \nabla \varphi_{n}\right\|_{q, Q_{R}} \\
& +\frac{c \rho^{\frac{N+2}{q}}}{(R-\rho)^{\frac{N+2}{2}}}\left(\left\|\nabla u_{n}\right\|_{2, Q_{R}}+\left\|\nabla v_{n}\right\|_{2, Q_{R}}\right) \\
\leq & c(q)\left\|\sigma_{n}\left(u_{n}\right) \varphi_{n} \nabla \varphi_{n}\right\|_{q, Q_{R}} \\
& +\frac{c \rho^{\frac{N+2}{q}}}{(R-\rho)^{\frac{N+2}{2}}}\left(\left\|\nabla u_{n}\right\|_{2, Q_{R}}+\left\|\sigma_{n}\left(u_{n}\right) \varphi_{n} \nabla \varphi_{n}\right\|_{2, Q_{R}}\right) .
\end{aligned}
$$

Define, for $k \in\{0,1,2, \ldots\}$,

$$
\rho_{k}=R-\frac{R}{2^{k+1}} .
$$

Set $\rho=\rho_{k}, R=\rho_{k}+\frac{1}{2}\left(\rho_{k+1}-\rho_{k}\right)=\frac{1}{2}\left(\rho_{k+1}+\rho_{k}\right)$ in (3.10) to obtain

$$
\left\|\nabla \varphi_{n}\right\|_{q, Q_{\rho}} \leq c(q)\left(\left\|\nabla u_{n}\right\|_{q, Q_{\frac{1}{2}\left(\rho_{k+1}+\rho_{k}\right)}}+c R^{\frac{N+2}{q}-1} 2^{k}\right) .
$$


Let $\rho=\frac{1}{2}\left(\rho_{k+1}+\rho_{k}\right), R=\rho_{k+1}$ in $(3.23)$ to get

$$
\begin{aligned}
\left\|\nabla u_{n}\right\|_{q, Q \frac{1}{2}\left(\rho_{k+1}+\rho_{k}\right)} \leq & c(q)\left\|\sigma_{n}\left(u_{n}\right)\right\|_{\infty, Q_{R}}\left\|\nabla \varphi_{n}\right\|_{q, Q_{\rho_{k+1}}} \\
& +c R^{\frac{N+2}{q}-\frac{N+2}{2}}\left(2^{\frac{N+2}{2}}\right)^{k} .
\end{aligned}
$$

Use this in (3.24) to obtain

$$
\begin{aligned}
\left\|\nabla \varphi_{n}\right\|_{q, Q_{\rho_{k}}} \leq & c(q)\left\|\sigma_{n}\left(u_{n}\right)\right\|_{\infty, Q_{R}}\left\|\nabla \varphi_{n}\right\|_{q, Q_{\rho_{k+1}}} \\
& +c\left(R^{\frac{N+2}{q}-1}+R^{\frac{N+2}{q}-\frac{N+2}{2}}\right)\left(2^{\frac{N+2}{2}}\right)^{k} .
\end{aligned}
$$

Thus, if

$$
c(q)\left\|\sigma_{n}\left(u_{n}\right)\right\|_{\infty, Q_{R}} \cdot 2^{\frac{N+2}{2}} \leq \frac{1}{2},
$$

we have

$$
\left\|\nabla \varphi_{n}\right\|_{q, Q_{\frac{R}{2}}} \leq c\left(R^{\frac{N+2}{q}-1}+R^{\frac{N+2}{q}-\frac{N+2}{2}}\right) .
$$

Recall that $\lim _{s \rightarrow \infty} \sigma(s)=0$ and $\sigma_{n}(s)=\sigma(s)+\frac{1}{n}$. We conclude that there is an $M_{0}>0$ such that (3.26) holds whenever $n$ is large enough and

$$
\text { ess } \inf _{Q_{R}} u_{n} \geq M_{0} \text {. }
$$

Now let $\left(x_{0}, t_{0}\right) \in Q_{T}$ and $c^{*}$ be given as in (2.1). Assume that there exists an $R_{1} \in\left(0, R_{0}\left(x_{0}, t_{0}\right) / 2\right)$ such that

$$
f_{Q_{2 R_{1}}\left(x_{0}, t_{0}\right)} u d x d t>c^{*}+M_{0}
$$

Then

$$
f_{Q_{2 R_{1}}\left(x_{0}, t_{0}\right)} u_{n} d x d t>c^{*}+M_{0}
$$

for $n$ sufficiently large, since a result in [X2] asserts that $u_{n} \rightarrow u$ strongly in $L^{2}\left(Q_{T}\right)$. By virtue of Theorem 2,

$$
\begin{aligned}
\text { ess } \inf _{Q_{R_{1}}} u_{n} & \geq f_{Q_{2 R_{1}}} u_{n} d x d t-c^{*} \\
& >M_{0} \text { for } n \text { sufficiently large. }
\end{aligned}
$$

This means that (3.27) holds for $R=R_{1}$ and $n$ large enough. Thus,

$$
\|\nabla \varphi\|_{q, Q_{\frac{R_{1}}{2}}} \leq c\left(q, R_{1}\right)
$$

for all $q>2$. Define

$$
\Gamma=\left\{(x, t) \in Q_{T}: \exists R \in\left(0, R_{0}(x, t) / 2\right): f_{Q_{R}(x, t)} u d x d t>c^{*}+M_{0}\right\} .
$$

Clearly, $\Gamma$ is open. For each $(x, t) \in \Gamma$, there is a $\rho_{0}>0$ such that $u$ is Hölder continuous on $Q_{\rho_{0}}(x, t)$ due to (3.29). We see that

$$
u \leq c^{*}+M_{0}
$$


a.e. on $Q_{T} \backslash \Gamma$. Now, let

$$
Q_{0}=\Gamma \cup \text { Interior of } Q_{T} \backslash \Gamma .
$$

The local Hölder continuity of $u$ on the interior of $Q_{T} \backslash \Gamma$ is due to a local version

of Theorem 7. This completes the proof.

\section{REFERENCES}

[AC] S.N. Antontsev and M. Chipot, The thermistor problem : existence, smoothness, uniqueness, and blow up, SIAM J. Math. Anal. 25 (1994), 1128-1156. MR 95f:35013

[BLP] A. Bensoussan, J.L. Lions, and G. Papanicolaou, Asymptotic Analysis for Periodic Structures, North-Holland Publ. Co., Amsterdam, 1978. MR 82h:35001

[C] X. Chen, Existence and regularity of solutions of a nonlinear degenerate elliptic system arising from a thermistor problem, J. Partial Differential Equations 7 (1994), 19-34. MR 95c:35078

[D] E. DiBenedetto, Degenerate Parabolic Equations, Springer-Verlag, New York, 1993. MR 94h:35130

[DM] E. DiBenedetto and J. Manfredi, On the higher integrability of the gradient of weak solutions of certain degenerate elliptic systems, Amer. J. Math. 115 (1993), 1107-1134. MR 94i: 35077

[FS] C. Fefferman and E.M. Stein, $H^{p}$ spaces of several variables, Acta Math. 129 (1972), 137-193. MR 56:6263

[G] M. Giaquinta, Multiple Integrals in the Calculus of Variations and Nonlinear Elliptic Systems, Princeton University Press, Princeton, New Jersey, 1983. MR 86b:49003

[LSU] O.A. Ladyzenskaja, V.A. Solonnikov, and N.N. Ural'ceva, Linear and Quasilinear Equations of Parabolic Type, Transl. Math. Mono., Vol. 23, A.M.S., Providence, Rhode Island, 1968. MR 39:3159

[K] R.V. Kohn, The method of partial regularity as applied to the Navier-Stokes equations, Seminar on Nonlinear Partial Differential Equations, S.S. Chern, Ed., Springer-Verlag, New York, 1984, 117-128. MR 85g:35005

[R] J.F. Rodrigues, Obstacle Problems in Mathematical Physics, North-Holland Math. Studies, Vol. 134, North-Holland, Amsterdam, 1987. MR 88d:35006

[SSX] P. Shi, M. Shillor, and X. Xu, Existence of a solution to the Stefan problem with Joule's heating, J. Differential Equations 105 (1993), 239-263. MR 94k:35338

[SW] P. Shi and S. Wright, Higher integrability of the gradient in linear elasticity, Math. Ann. 299 (1994), 435-448. MR 95f:35034

[XA] H. Xie and W. Allegretto, $C^{\alpha}(\bar{\Omega})$-solution of a class of nonlinear degenerate elliptic systems arising in the thermistor problem, SIAM J. Math. Anal. 22(1991), 1491-1499. MR 92m:35099

[X1] X. Xu, A strongly degenerate system involving an equation of parabolic type and an equation of elliptic type, Comm. Partial Differential Equations 18 (1993), 199-213. MR 95c:35182

[X2] X. Xu, A $p$-Laplacian problem in $L^{1}$ with nonlinear boundary conditions, Comm. Partial Differential Equations 19 (1994), 143-176. MR 95b:35075

[Y] G. Yuan, Regularity of solutions of the nonstationary thermistor problem, Applicable Anal. 53(1994), 149-169. CMP 1996:9

[YL] G. Yuan and Z. Liu, Existence and uniqueness of the $C^{\alpha}$-solution for the thermistor problem with mixed boundary value, SIAM J. Math. Anal. 25 (1994), 1157-1166. MR 95f:35097

Department of Mathematics and Statistics, Mississippi State University, Mississippi State, Mississippi 39762

E-mail address: xxu@math.msstate.edu 